\title{
Modal Tilt/Translate Control and Stability of a Rigid Rotor with Gyroscopics on Active Magnetic Bearings
}

\author{
Timothy Dimond, ${ }^{1}$ Paul Allaire, ${ }^{1}$ Simon Mushi, ${ }^{2}$ Zongli Lin, ${ }^{2}$ and Se Young Yoon ${ }^{2}$ \\ ${ }^{1}$ Rotating Machinery and Controls Laboratory, Department of Mechanical and Aerospace Engineering, University of Virginia, \\ 122 Engineer's Way, Charlottesville, VA 22904, USA \\ ${ }^{2}$ Charles L. Brown Department of Electrical and Computer Engineering, University of Virginia, Charlottesville, VA 22904, USA
}

Correspondence should be addressed to Timothy Dimond, twd5c@virginia.edu

Received 25 August 2011; Revised 3 January 2012; Accepted 8 January 2012

Academic Editor: Jerzy Sawicki

Copyright (c) 2012 Timothy Dimond et al. This is an open access article distributed under the Creative Commons Attribution License, which permits unrestricted use, distribution, and reproduction in any medium, provided the original work is properly cited.

Most industrial rotors supported in active magnetic bearings (AMBs) are operated well below the first bending critical speed. Also, they are usually controlled using proportional, integral and derivative controllers, which are set up as modally uncoupled parallel and tilt rotor axes. Gyroscopic effects create mode splitting and a speed-dependent plant. Two AMBs with four axes of control must simultaneously control and stabilize the rotor/AMB system. Various analyses have been published considering this problem for different rotor/AMB configurations. There has not been a fully dimensionless analysis of these rigid rotor AMB systems. This paper will perform this analysis with a modal PD controller in terms of translation mode and tilt mode dimensionless eigenvalues and eigenvectors. The number of independent system parameters is significantly reduced. Dimensionless PD controller gains, the ratio of rotor polar to transverse moments of inertia and a dimensionless speed ratio are used to evaluate a fully general system stability rigid rotor analysis. An objective of this work is to quantify the effects of gyroscopics on rigid rotor AMB systems. These gyroscopic forces reduce the system stability margin. The paper is also intended to help provide a common framework for communication between rotating machinery designers and controls engineers

\section{Introduction}

Active magnetic bearings (AMBs) have been in use since the 1930s and are an alternative to fluid film bearings in the support and stabilization of rotating machinery [1]. Active magnetic bearings are a noncontact method of supporting a rotor and offer distinct advantages due to elimination of lubrication systems, reduced parasitic losses, and reduced wear. However, implementation of active control in AMB systems adds complexity to the overall rotordynamics, including the stability of the rotor. The AMBs are open-loop unstable, and the presence of gyroscopics further complicates the control problem.

Many AMB rotor applications operate well below the first bending natural frequency of the rotor, allowing for treatment of the rotor as a rigid body. This greatly simplifies the problem as the rotor can be represented with five degrees of freedom. Gosiewski and Falkowski [2] developed a control law based upon a rigid rotor model for an AMB-suspended gyroscope for space applications. A linearized AMB model was employed and equations of motion were obtained for the gyroscope undergoing aircraft motions.

Matsumura et al. [3] further developed the rigid rotor second-order equations of motion, including gyroscopic effects, based upon rotations about the inertia axes. They converted the system to state space form and used an output regulator to stabilize the rotor/AMB system. Then, they employed a feed-forward controller to minimize unbalance response. A similar study was presented by Mizuno and Higuchi [4]. Kim and Han [5] presented the second-order equations of motion for a rigid rotor/AMB system and converted them to a dimensionless state space form. They considered a general servocontroller and a centralized PID controller, evaluating disturbance rejection, reference tracking, and robustness. However, the authors did not consider the industry most common modal tilt/translate control or the associated 
PD control and closed loop system properties including dimensionless gyroscopic effects relative to dimensionless tilt/ translate natural frequencies.

Lottin et al. [6] presented the nonlinear control of a relatively long, thin rigid rotor on two AMBs with digital implementation. No bias current was employed to reduce the AMB power consumption. The control algorithm did not take into account the gyroscopic coupling. It was found not to be significant for this long thin rotor, which was operating at a low speed in the reported experimental tests. One of the major issues examined in this paper was the importance of the gyoscopic effects on the rotor and some estimate of whether the controller needs to go to significant lengths to take them into account.

Cao et al. [7] analyzed a flywheel battery with a rigid rotor on AMBs. With the flywheel disk attached, it is similar to a disk rotor. The stated purpose of the paper is "a decoupling approach for the nonlinear model of the flywheel energy storage device supported by active magnetic bearings such that the instability brought on by gyroscopic effects can be overcome." The decoupling approach involved a nonlinear model of the control system based upon dynamic feedback linearization. The strongly coupled rotor motion was reduced to five normalization subsystems including four radial displacements and the rotor speed. These linear subsystems are completely decoupled from one another and linearized controllers were developed. The rotor was successfully levitated. However, the authors note that the decoupling control was found to be very sensitive to variations between the rotor model and the actual rotor. Based upon the reports of large efforts made by various researchers, the study in the present paper to quantify the gyroscopic effects on dimensionless properties of rigid rotors in AMBs is quite worthwhile.

Ho et al. [8] investigated the effect of an active thrust bearing on the stability of a rigid rotor supported on AMBs. Decentralized PID controllers were implemented. Due to tilting imposed by the thrust bearing, it was demonstrated that the thrust bearing reduced the stability of the tilt modes.

Zhao et al. [9] developed PD control of a rigid rotor supported by AMBs for a momentum wheel application. The momentum wheel is used in spacecraft to actuate the attitude control system. A Linear Quadratic Regulator (LQR) controller was used to account for the large gyroscopic coupling imposed by a flywheel. The LQR algorithm was also implemented by Pilat [10] in a simulation study. Pilat considered a nonlinear inductance in modeling the actuator forces and then linearized the system about the operating point. A SISO controller was then implemented on each AMB axis.

Rigid rotor applications have also been considered in studies of unbalance compensation. Löwis and Rudolph [11] developed an adaptive algorithm for unbalance compensation. The control law included PD control for position and proportional control of the unbalance level, resulting in rotation about the inertial center. Since the unbalance level was unknown, an estimator of the unbalance level was used in the control law. The stability algorithm was demonstrated using a rigid rotor model with gyroscopic effects included.
Li et al. [12] developed an unbalance compensation routine for rigid rotors that included a compensation term for the unbalance and an estimate of the ratio of open-loop stiffness to current gain in the control law. The combination of the two parameters resulted in rotation about the inertial center with proper pole placement. The control laws were based on SISO controllers. Gyroscopics were not considered.

The asymptotic stability of rigid rotors supported in AMBs has also been an area of study. Recent work in this area includes the study by Jeon et al. [13]. They investigated the effect of sensor noncollocation on the overall stability of a rigid rotor supported in AMBs. Using a rigid rotor model with gyroscopic effects included and a PD controller, the system eigenvalues were derived in terms of the bearing coordinates and a factor representing the distance between the sensors and the actuators. Based on Lyapunov and Kelvin-Tait-Chetaev stability criteria, it was shown that sensor noncollocation can result in instability of the rigid rotor. The stability region was improved by treating sensor noncollocation as a time delay in the system model.

Gosiewski [14] examined stability of an AMB supported rigid rotor with $\mathrm{PD}$ control and noncollocated sensors. The overall state-space system model with eight degrees of freedom was reduced to four degrees of freedom. The stability of the system was assessed using the Hurwitz stability criterion and with root locus plots. The effect of sensor noncollocation was mitigated by a coordinate transformation between the sensor location and the actuator location. Investigation of a simulated rotor indicated that the overall system stability was linked to location of a noncollocated sensor relative to the node point in the tilt mode.

The current work considers a rigid rotor supported in active magnetic bearings with gyroscopic effects included. The model has been previously presented by Larsonneur in [15]. The PD control in this paper's model is based on the mass center coordinates of the shaft, with rotation about the principal axis most closely parallel to the axial direction. From this model, an equivalent four degree of freedom system representing translate and tilt modes including damping and gyroscopic effects is determined. The equations of motion are then nondimensionalized. A new analytical solution for the damped nondimensional eigenvalues for both translate and tilt modes is obtained due to modal decoupling in terms of the zero-rotational speed damping ratio and natural frequencies, the nondimensional rotational frequency, and the ratio of polar to transverse mass moment of inertia.

The effect of gyroscopics and damping on the tilt mode natural frequencies and stability margin is calculated. Many AMB supported rotors are relatively short, but others are relatively long and thin. Both types are considered in this paper. One objective of the study is to determine what conditions are relatively strongly affected by gyroscopics and which are not.

The stability margin is expressed in terms of the logarithmic decrement, which allows direct comparisons to typical stability assessments of rotors supported in fluid bearings and is, therefore, beneficial for the turbomachinery community. A previous study by the authors [16] demonstrated the similarities in unbalance response between fluid film 


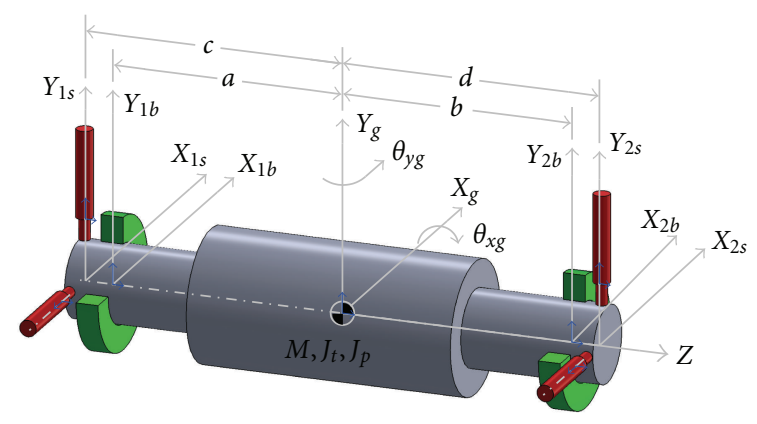

FIGURE 1: Rigid rotor free body diagram.

bearing and active magnetic bearing systems. The present study is also intended to help provide a common framework to facilitate communication between machine designers, typically mechanical engineers, and controls designers, often electrical engineers.

\section{Analysis}

First, the open-loop characteristics of a rigid rotor model with gyroscopics on AMBs is considered. Then, an active magnetic bearing with $\mathrm{PD}$ control and compensation for the open-loop stiffness is considered, and the resulting free-vibration rotor model is presented. The translate and tilt mode solutions are obtained, and the effect of the gyroscopics on the logarithmic decrement and the undamped natural frequency of the tilt modes is examined in detail.

2.1. Rotor Model. For the purposes of this paper, the rotor is modeled as rigid with gyroscopic effects included. In effect, the rotor is considered to be running well below its first bending critical speed. These assumptions apply to a wide class of machines including motors, flywheels, and gyroscopes supported on active magnetic bearings. A free body diagram of the rigid rotor is shown in Figure 1.

The equation of motion for the rotor supported in magnetic bearings is then

$$
\mathbf{M} \ddot{\mathbf{u}}_{g}+\Omega \mathbf{G} \dot{\mathbf{u}}_{g}=\mathbf{f}_{g, \mathrm{AMB}},
$$

where $\Omega$ is the rotational speed of the shaft, $\mathbf{f}_{g, \mathrm{AMB}}=$ $\left[\begin{array}{llll}F_{x g} & F_{y g} & M_{x g} & M_{y g}\end{array}\right]^{T}$ are the magnetic forces and moments about the rotor gravity center produced by the magnetic bearings, and the vector $\mathbf{u}=\left[\begin{array}{llll}x_{g} & y_{g} & \theta_{x g} & \theta_{y g}\end{array}\right]^{T}$ indicates rotor translations and transverse rotations about the center of gravity (c. g.). The inertia matrix $\mathbf{M}$ and the gyroscopic matrix $\mathbf{G}$ are defined as

$$
\mathbf{M}=\left[\begin{array}{cccc}
M & 0 & 0 & 0 \\
0 & M & 0 & 0 \\
0 & 0 & J_{t} & 0 \\
0 & 0 & 0 & J_{t}
\end{array}\right] ; \quad \mathbf{G}=\left[\begin{array}{cccc}
0 & 0 & 0 & 0 \\
0 & 0 & 0 & 0 \\
0 & 0 & 0 & J_{p} \\
0 & 0 & -J_{p} & 0
\end{array}\right],
$$

where $M$ is the mass of the rotor, $J_{t}$ is the transverse mass moment of inertia about the c. g., and $J_{p}$ is the polar mass moment of inertia about the c. g. Equation (1) indicates that the only external forces acting on the rotor are due to the active magnetic bearings. The rotor model also appears in [15], although some differences in signs of displacements occur due to the selection of the coordinate system in this paper.

2.2. Coordinate Transformations. The bearings are acting at positions $1 b$ and $2 b$ in Figure 1 . It is desired in this paper to keep the analysis using the $\mathrm{c}$. g. coordinate system as the mass and gyroscopic matrices are simpler. Thus, it is necessary to transform from the bearing coordinates to the gravity-center coordinates since the equations of motion are formulated in the gravity-center coordinate system. The transform from bearing coordinates to gravity-center coordinates is given by

$$
\underbrace{\left\{\begin{array}{c}
x_{1 b} \\
y_{1 b} \\
x_{2 b} \\
y_{2 b}
\end{array}\right\}}_{\mathbf{u}_{b}}=\underbrace{\left[\begin{array}{cccc}
1 & 0 & 0 & -a \\
0 & 1 & a & 0 \\
1 & 0 & 0 & b \\
0 & 1 & -b & 0
\end{array}\right]}_{\mathbf{Q}_{b}} \underbrace{\left\{\begin{array}{c}
x_{g} \\
y_{g} \\
\theta_{x g} \\
\theta_{y g}
\end{array}\right\}}_{\mathbf{u}_{g}},
$$

where $x_{1 b}, y_{1 b}, x_{2 b}$, and $y_{2 b}$ refer to the rotor translations at the bearings, and $a$ and $b$ are the axial distances from each bearing to the rotor gravity center.

The sensors are in general not collocated with the bearing center of action. To account for this, an additional transform is required to move from the sensors to the gravity center coordinate system. The measurement locations are used for design of the feedback controller described in Section 2.3.2. Analogous to the bearing coordinate transformation, the sensor coordinate transformation is given by

$$
\left\{\begin{array}{l}
x_{1 s} \\
y_{1 s} \\
x_{2 s} \\
y_{2 s}
\end{array}\right\}=\underbrace{\left[\begin{array}{cccc}
1 & 0 & 0 & -c \\
0 & 1 & c & 0 \\
1 & 0 & 0 & d \\
0 & 1 & -d & 0
\end{array}\right]}_{\mathbf{u}_{s}} \underbrace{\left\{\begin{array}{c}
x_{g} \\
y_{g} \\
\theta_{x g} \\
\theta_{y g}
\end{array}\right\}}_{\mathbf{Q}_{s}},
$$

where $x_{1 s}, y_{1 s}, x_{2 s}$, and $y_{2 s}$ are the sensor axial locations, and $c$ and $d$ are the axial distances from the sensors to the rotor gravity center.

2.3. AMB Model. As is standard in active magnetic bearing analyses, the magnetic forces are linearized about the operating point, giving the force for each $\mathrm{AMB}$ axis in terms of rotor displacement and perturbation current as

$$
\begin{aligned}
& F_{x b} \approx k_{i} i_{p}+k_{h} x, \\
& F_{y b} \approx k_{i} i_{p}+k_{h} y,
\end{aligned}
$$

where $i_{p}$ is the perturbation current, $k_{i}$ represents the current gain, and $k_{h}$ represents the open-loop stiffness. 
When the $x$ and $y$ directions are considered for each magnetic bearing in Figure 1, matrix equations can be formed for the open loop bearing characteristics in terms of the bearing coordinates. The resulting force model is

$$
\mathbf{f}_{b, \mathrm{AMB}}=\mathbf{K}_{\mathbf{h}} \mathbf{u}_{\mathbf{b}}+\mathbf{K}_{\mathbf{i}} \mathbf{i}_{\mathbf{p}},
$$

where $\mathbf{u}_{\mathbf{b}}=\left[\begin{array}{llll}x_{1 b} & y_{1 b} & x_{2 b} & y_{2 b}\end{array}\right]^{T}$ and $\mathbf{i}_{\mathbf{p}}=\left[\begin{array}{llll}i_{p, x 1 b} & i_{p, y 1 b} & i_{p, x 2 b} & i_{p, x 2 b}\end{array}\right]^{T}$. The resulting matrix of cur-rent gains $\mathbf{K}_{\mathbf{i}}$ for the perturbation currents is given by $k_{i} \mathbf{I}$, and the open-loop stiffness matrix $\mathbf{K}_{\mathbf{h}}$ is given by $k_{h} \mathbf{I}$, where $\mathbf{I}$ is the identity matrix.

2.3.1. Open-Loop Characteristics. The active magnetic bearings considered only apply forces at the locations indicated in Figure 1. The net forces and moments acting at the gravity center of the rotor are then obtained by a force and moment balance. The forces in moments at the gravity center due to the AMBs are expressed in matrix form as

$$
\underbrace{\left\{\begin{array}{c}
F_{x g} \\
F_{y g} \\
M_{x g} \\
M_{y g}
\end{array}\right\}}_{\mathbf{f}_{g, \mathrm{AMB}}}=\underbrace{\left[\begin{array}{cccc}
1 & 0 & 1 & 0 \\
0 & 1 & 0 & 1 \\
0 & a & 0 & -b \\
-a & 0 & b & 0
\end{array}\right]}_{\mathbf{Q}_{b}^{T}} \underbrace{\left\{\begin{array}{c}
F_{x 1 b} \\
F_{y 1 b} \\
F_{x 2 b} \\
F_{y 2 b}
\end{array}\right\}}_{\mathbf{f}_{b, \mathrm{AMB}}} .
$$

The coordinate transformation represented by (7) indicates that the tilt and translate modes are coupled by the magnetic bearing open loop characteristics for rigid rotor applications. It should be noted that the force transformation matrix is the transpose of the coordinate transformation matrix described in (3). Using the transformation defined by (7), the rotor model described by (1) becomes

$$
\mathbf{M} \ddot{\mathbf{u}}_{g}+\Omega \mathbf{G} \dot{\mathbf{u}}_{g}=\mathbf{Q}_{b}^{T} \mathbf{f}_{b, \mathrm{AMB}} .
$$

Then, by substitution of (3) and (6) into (8), the resulting $\mathrm{AMB}$ open-loop equation of motion is then

$$
\mathbf{M} \ddot{\mathbf{u}}_{g}+\Omega \mathbf{G} \dot{\mathbf{u}}_{g}-\mathbf{Q}_{b}^{T} \mathbf{K}_{\mathbf{h}} \mathbf{Q}_{b} \mathbf{u}_{g}=\mathbf{Q}_{b}^{T} \mathbf{K}_{\mathbf{i}} \mathbf{i}_{p} .
$$

Equation (9) represents an unstable system [15]. The negative feedback controller described in Section 2.3.2 is used to stabilize the system and eliminate the coupling of the tilting and translating modes imposed on the system by (7).

2.3.2. PD Control Design. For this study, it is desired to express the equations of motion in terms of the gravity centered coordinates and the control gains. In terms of the sensor coordinates referred to the gravity center coordinates, the negative feedback control law for the active magnetic bearings is [15]

$$
\begin{aligned}
\mathbf{i}_{\mathbf{p}}= & \underbrace{-\mathbf{K}_{\mathbf{i}}^{-1} \mathbf{Q}_{b}^{-T} \mathbf{K}_{\mathbf{P}} \underbrace{\mathbf{Q}_{s}^{-1} \mathbf{u}_{s}}_{\mathbf{u}_{g}}}_{\text {Proportional Term }}-\underbrace{\mathbf{K}_{\mathbf{i}}^{-1} \mathbf{Q}_{b}^{-T} \mathbf{K}_{\mathbf{D}} \underbrace{\mathbf{Q}_{s}^{-1} \dot{\mathbf{u}}_{s}}_{\dot{\mathbf{u}}_{g}}}_{\text {Derivative Term }} \\
& -\underbrace{\mathbf{K}_{\mathbf{i}}^{-1} \mathbf{K}_{\mathbf{h}} \mathbf{Q}_{b} \underbrace{\mathbf{Q}_{s}^{-1} \mathbf{u}_{s}}_{\mathbf{u}_{g}}}_{\text {Compensator Term }},
\end{aligned}
$$

where $\mathbf{K}_{\mathbf{P}}$ is the proportional control gain matrix and $\mathbf{K}_{\mathbf{D}}$ is the derivative control gain matrix. The control law is a modal PD controller with a compensation term for the openloop stiffness characteristics of the AMBs. It may be noted that there is no $x-y$ coordinate coupling in the controller to account for gyroscopic forces.

Substituting (10) into (9), the closed-loop AMB-rotor system of equations becomes

$$
\begin{aligned}
& \mathbf{M} \ddot{\mathbf{u}}_{g}+\Omega \mathbf{G} \dot{\mathbf{u}}_{g}-\mathbf{Q}_{b}^{T} \mathbf{K}_{\mathbf{h}} \mathbf{Q}_{b} \mathbf{u}_{g} \\
&=-\mathbf{Q}_{b}^{T} \mathbf{K}_{\mathbf{i}}\left[\mathbf{K}_{\mathbf{i}}^{-1} \mathbf{Q}_{b}^{-T} \mathbf{K}_{\mathbf{P}} \mathbf{Q}_{s}^{-1} \mathbf{u}_{s}+\mathbf{K}_{\mathbf{i}}^{-1} \mathbf{Q}_{b}^{-T} \mathbf{K}_{\mathbf{D}} \mathbf{Q}_{s}^{-1} \dot{\mathbf{u}}_{s}\right. \\
&\left.\quad+\mathbf{K}_{\mathbf{i}}^{-1} \mathbf{K}_{\mathbf{h}} \mathbf{Q}_{b} \mathbf{Q}_{s}^{-1} \mathbf{u}_{s}\right] .
\end{aligned}
$$

Then, the equations of motion including the controller in terms of the center of gravity coordinates reduce to [15]

$$
\mathbf{M} \ddot{\mathbf{u}}_{g}+\left(\Omega \mathbf{G}+\mathbf{K}_{\mathbf{D}}\right) \dot{\mathbf{u}}_{g}+\mathbf{K}_{\mathbf{P}} \mathbf{u}_{g}=0
$$

Implicit in (12) are the perturbation currents and the current gain that are typical in linearized AMB control. The diagonal derivative control matrix $\mathbf{K}_{\mathbf{D}}$ and the diagonal proportional matrix $\mathbf{K}_{\mathbf{P}}$ are defined as

$$
\mathbf{K}_{\mathbf{D}}=\left[\begin{array}{cccc}
k_{d, t} & 0 & 0 & 0 \\
0 & k_{d, t} & 0 & 0 \\
0 & 0 & k_{d, r} & 0 \\
0 & 0 & 0 & k_{d, r}
\end{array}\right] ; \quad \mathbf{K}_{\mathbf{P}}=\left[\begin{array}{cccc}
k_{p, t} & 0 & 0 & 0 \\
0 & k_{p, t} & 0 & 0 \\
0 & 0 & k_{p, r} & 0 \\
0 & 0 & 0 & k_{p, r}
\end{array}\right] \text {, }
$$

where $k_{d, t}$ and $k_{d, r}$ represent derivative gains for translational and rotational degrees of freedom, respectively. The variables $k_{p, t}$ and $k_{p, r}$ represent proportional gains for translational and rotational degrees of freedom. In this formulation, the derivative gain control matrix acts as damping, and the proportional gain control matrix acts as stiffness. The advantage to expressing the control law as (12) is that the benefits of multiple-input multiple-output (MIMO) control are obtained in a form that is similar to single-input single-output (SISO) control. This allows for a more intuitive "feel" for the control action on the rotor [15] and makes the model more accessible for mechanical engineers that do not work with control theory on a regular basis.

Since only rigid-body modes are considered, the damping level represented by $k_{d, t}$ can be chosen to be any positive number without affecting the system stability [15]. This paper considers $k_{d, t}$ values that result in a damping ratio of less than 1 , which is typical for PD controls when rise time and settling time are design considerations. The proportional gain $k_{p, t}$ is generally chosen such that the ratio of closed loop stiffness to open loop stiffness $k_{p, t} / k_{h}$ is between 1 to 3 [15].

Sensor noncollocation is not much on an issue for rigid rotors. A well-behaved coordinate transformation can always be found for relating the sensor signals to the bearing locations. Thus, this topic is not discussed further. 
2.4. Translating Mode Damped Eigenvalues. Another advantage to (12) is that the translating modes are now decoupled from the tilt modes. The translating modes are described by the uncoupled translational degrees of freedom or

$$
\left[\begin{array}{cc}
M & 0 \\
0 & M
\end{array}\right]\left\{\begin{array}{c}
\ddot{x}_{g} \\
\ddot{y}_{g}
\end{array}\right\}+\left[\begin{array}{cc}
k_{d, t} & 0 \\
0 & k_{d, t}
\end{array}\right]\left\{\begin{array}{c}
\dot{x}_{g} \\
\dot{y}_{g}
\end{array}\right\}+\left[\begin{array}{cc}
k_{p, t} & 0 \\
0 & k_{p, t}
\end{array}\right]\left\{\begin{array}{l}
x_{g} \\
y_{g}
\end{array}\right\}=\left\{\begin{array}{l}
0 \\
0
\end{array}\right\} .
$$

The damped eigenvalues of the translating modes are not a function of the running speed due to the decoupling. Taking the horizontal translation as an example, the equation of motion is

$$
M \ddot{x}_{g}+k_{d, t} \dot{x}_{g}+k_{p, t} x_{g}=0 .
$$

Equation (15) can be placed in standard form as

$$
\ddot{x}_{g}+2 \zeta_{t} \omega_{n, t} \dot{x}_{g}+\omega_{n, t}^{2} x_{g}=0 \text {, }
$$

where $\zeta_{t}$ represents the translate modal damping ratio. The undamped translational natural frequency $\omega_{n, t}$ is used to nondimensionalize the damped natural frequency. Assuming that the transverse damping ratio $\zeta_{t}$ is less than one, the complex solution for the dimensionless damped natural frequency $\bar{s}_{t}$ is

$$
\bar{s}_{t}=-\zeta_{t} \pm j \sqrt{1-\zeta_{t}^{2}}
$$

The vertical translation solution is identical. This is the dimensionless translating eigenvalue solution.

2.5. Tilting-Mode-Damped Eigenvalues. The tilt modes are represented by the rotational degrees of freedom about the transverse axes, and the equations of motion are

$$
\begin{gathered}
{\left[\begin{array}{ll}
J_{t} & 0 \\
0 & J_{t}
\end{array}\right]\left\{\begin{array}{l}
\ddot{\theta}_{x g} \\
\ddot{\theta}_{y g}
\end{array}\right\}+\left(\Omega\left[\begin{array}{cc}
0 & J_{p} \\
-J_{p} & 0
\end{array}\right]+\left[\begin{array}{cc}
k_{d, r} & 0 \\
0 & k_{d, r}
\end{array}\right]\right)\left\{\begin{array}{c}
\dot{\theta}_{x g} \\
\dot{\theta}_{y g}
\end{array}\right\}} \\
+\left[\begin{array}{cc}
k_{p, r} & 0 \\
0 & k_{p, r}
\end{array}\right]\left\{\begin{array}{l}
\theta_{x g} \\
\theta_{y g}
\end{array}\right\}=\left\{\begin{array}{l}
0 \\
0
\end{array}\right\} .
\end{gathered}
$$

It should be noted that this system represents an extension of the undamped rigid rotor analysis found in many textbooks [17-19]. The effect of gyroscopics and the PD control action are now considered for the natural frequencies corresponding to the tilt modes. A solution of the form $\theta_{x}=$ $\Theta_{x} e^{s t}, \theta_{y}=\Theta_{y} e^{s t}$, is assumed for the analysis. Equation (18) then becomes:

$$
\left[\begin{array}{cc}
s^{2} J_{t}+s k_{d, r}+k_{p, r} & s \Omega J_{p} \\
-s \Omega J_{p} & s^{2} J_{t}+s k_{d, r}+k_{p, r}
\end{array}\right]\left\{\begin{array}{l}
\Theta_{x g} \\
\Theta_{y g}
\end{array}\right\}=\left\{\begin{array}{l}
0 \\
0
\end{array}\right\} .
$$

The system-damped eigenvalues are then found by setting the determinant of the impedance matrix equal to zero or

$$
\left|\begin{array}{cc}
s^{2} J_{t}+s k_{d, r}+k_{p, r} & s \Omega J_{p} \\
-s \Omega J_{p} & s^{2} J_{t}+s k_{d, r}+k_{p, r}
\end{array}\right|=0 .
$$

The resulting characteristic equation for the tilt modes is then

$$
\left(s^{2} J_{t}+s k_{d, r}+k_{p, r}\right)^{2}+s^{2}\left(\Omega J_{p}\right)^{2}=0 .
$$

Equation (21) can then be factored into two characteristic equations that have four total roots. These four roots correspond to four tilt modes, two forward and two backward. The two characteristic equations are then

$$
\begin{aligned}
& s^{2} J_{t}+s\left(k_{d, r}+j \Omega J_{p}\right)+k_{p, r}=0, \\
& s^{2} J_{t}+s\left(k_{d, r}-j \Omega J_{p}\right)+k_{p, r}=0 .
\end{aligned}
$$

Placing (22) in standard form yields

$$
\begin{aligned}
& s^{2}+s\left(2 \zeta_{r, 0} \omega_{r n, 0}+j \Omega P\right)+\omega_{r n, 0}^{2}=0, \\
& s^{2}+s\left(2 \zeta_{r, 0} \omega_{r n, 0}-j \Omega P\right)+\omega_{r n, 0}^{2}=0 .
\end{aligned}
$$

Here, we have defined the tilt mode damping ratio $\zeta_{r, 0}$ and the tilt mode natural frequency $\omega_{r n, 0}$ to be the values obtained at zero rotational speed. The ratio of polar inertia to transverse inertia is also defined as $P=J_{p} / J_{t}$. For cylindrical sections, $P$ can assume values from 0 to 2 . A ratio of $P=0$ corresponds to an infinitely long cylindrical rotor, and a ratio of $P=2$ corresponds to a thin disk rotor.

Using the zero-rotational speed-undamped natural frequency $\omega_{r n, 0}$ to nondimensionalize the damped natural frequencies, the characteristic equations take the form:

$$
\bar{s}^{2}+\bar{s}\left(2 \zeta_{r, 0}+j \bar{\Omega} P\right)+1=0
$$

$$
\bar{s}^{2}+\bar{s}\left(2 \zeta_{r, 0}-j \bar{\Omega} P\right)+1=0 .
$$

Considering (24) first, the solutions for $\bar{s}_{r 1, r 2}$ using the quadratic formula is

$$
\bar{s}_{r 1, r 2}=\frac{-2 \zeta_{r, 0}-j \bar{\Omega} P \pm \sqrt{\left(2 \zeta_{r, 0}+j \bar{\Omega} P\right)^{2}-4}}{2} .
$$

For this solution, we assume that the controller derivative gain is such that the tilt modes are underdamped. After some rearrangement, equation (26) becomes

$$
\bar{s}_{r 1, r 2}=-\left(\zeta_{r, 0}+j \frac{\bar{\Omega} P}{2}\right) \pm j \sqrt{1-\zeta_{r, 0}^{2}+\left(\frac{\bar{\Omega} P}{2}\right)^{2}-j \zeta_{r, 0} \bar{\Omega} P} .
$$

Using a solution for the square root of a complex number $[20,21]$, the first two roots of the characteristic equation, represented by (27), become 


$$
\begin{aligned}
\bar{s}_{r 1, r 2}= & -\zeta_{r, 0} \pm \sqrt{\frac{1}{2}} \sqrt{\sqrt{\left[1-\zeta_{r, 0}^{2}+\left(\frac{\bar{\Omega} P}{2}\right)^{2}\right]^{2}+\left(\zeta_{r, 0} \bar{\Omega} P\right)^{2}}-1+\zeta_{r, 0}^{2}-\left(\frac{\bar{\Omega} P}{2}\right)^{2}} \\
& +j\left[-\frac{\bar{\Omega} P}{2} \pm \sqrt{\frac{1}{2}} \sqrt{\sqrt{\left[1-\zeta_{r, 0}^{2}+\left(\frac{\bar{\Omega} P}{2}\right)^{2}\right]^{2}+\left(\zeta_{r, 0} \bar{\Omega} P\right)^{2}}+1-\zeta_{r, 0}^{2}+\left(\frac{\bar{\Omega} P}{2}\right)^{2}}\right]
\end{aligned}
$$

Using a similar procedure for (25), the remaining solutions

$\bar{s}_{r 3, r 4}$ are

$$
\begin{aligned}
\bar{s}_{r 3, r 4}= & -\zeta_{r, 0} \mp \sqrt{\frac{1}{2}} \sqrt{\sqrt{\left[1-\zeta_{r, 0}^{2}+\left(\frac{\bar{\Omega} P}{2}\right)^{2}\right]^{2}+\left(\zeta_{r, 0} \bar{\Omega} P\right)^{2}}-1+\zeta_{r, 0}^{2}-\left(\frac{\bar{\Omega} P}{2}\right)^{2}} \\
& +j\left[\frac{\bar{\Omega} P}{2} \pm \sqrt{\frac{1}{2} \sqrt{\sqrt{\left[1-\zeta_{r, 0}^{2}+\left(\frac{\bar{\Omega} P}{2}\right)^{2}\right]^{2}+\left(\zeta_{r, 0} \bar{\Omega} P\right)^{2}}+1-\zeta_{r, 0}^{2}+\left(\frac{\bar{\Omega} P}{2}\right)^{2}}} .\right.
\end{aligned}
$$

When the damping ratio is zero, (28) and (29) reduce to

$$
\begin{gathered}
\bar{s}_{r 1, r 2}=-j\left[\frac{\bar{\Omega} P}{2} \mp \sqrt{\left(\frac{\bar{\Omega} P}{2}\right)^{2}+1}\right], \\
\bar{s}_{r 3, r 4}=j\left[\frac{\bar{\Omega} P}{2} \pm \sqrt{\left(\frac{\bar{\Omega} P}{2}\right)^{2}+1}\right],
\end{gathered}
$$

which is the purely complex undamped solution presented in several texts on rotordynamics, for example, [17-19].

2.6. Natural Frequencies, Damping Ratios, and Log Decrements. The four damped tilting mode solutions to $\bar{s}$ are now in the form $p+j q$. From these relations, the effective tilt mode damping ratio $\zeta_{r}$ at a nondimensional rotational speed $\bar{\Omega}$ is given by

$$
\zeta_{r}=-\frac{p / q}{\sqrt{1+(p / q)^{2}}} .
$$

The undamped tilt mode dimensionless natural frequency $\bar{\omega}_{n}$ is then

$$
\bar{\omega}_{r n}=-\frac{p}{\zeta_{r}}
$$

Rotordynamic analyses often use the logarithmic decrement $\delta$ instead of the damping ratio to assess the stability margin and the amount of damping in the modes. The logarithmic decrement is directly related to the damping ratio and is given by:

$$
\delta=-\frac{2 \pi \zeta_{r}}{\sqrt{1-\zeta_{r}^{2}}}
$$

\section{Numerical Examples}

To demonstrate the analyses above, several examples are provided. A Campbell diagram is presented for the undamped eigenvalues for various values of $P$. A Campbell diagram is also presented for various zero-speed damping ratios assuming $P=0.5$ to show the sensitivity of the undamped natural frequencies to applied damping and gyroscopics. Several plots of logarithmic decrement for different zerospeed damping ratios to show the sensitivity of the stability margin to the gyroscopics.

3.1. Campbell Diagrams. First, the Campbell diagram arising from (30) and (31) is considered. This results in four tilt modes, two forward and two backward. Only some of the forward tilt modes can be excited by unbalance depending on the value of $P$, as demonstrated in Figure 2 and depending on the unbalance distribution. However, unmodeled system dynamics, including both forward and backward modes, can 


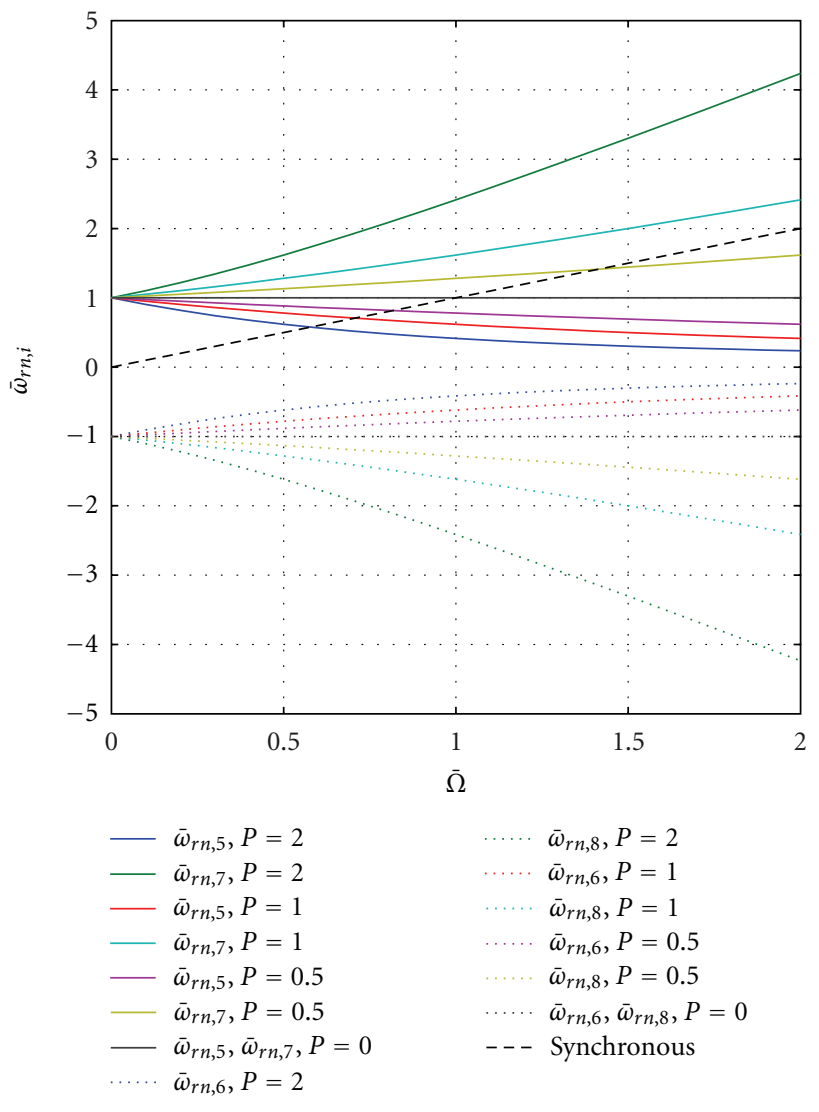

FIgURE 2: Rigid rotor Campbell diagram, tilt modes, $\zeta_{r, 0}=0$.

be excited by the AMBs if the modal frequency is within the controller bandwidth [22]. This is a significant difference from rotor systems supported on fluid film bearings. It is, therefore, important to consider all the modes within the controller bandwidth in the AMB controller design.

The next Campbell diagram is based on the undamped natural frequency, (33), for typical zero-speed damping ratios and a $P$ value of 0.5 . In Figure 3 , it is seen that the undamped tilt natural frequencies are not very sensitive to the zero-speed damping ratio. The change in natural frequency from $\zeta_{r, 0}=0$ to $\zeta_{r, 0}=0.7$ is less than ten percent for all four modes.

3.2. Effects of Rotational Speed on Stability Margin. The rotor/AMB system does not explicitly have a component designed to control gyroscopics. However, the rotor/AMB system is stable. This section discusses the reduction in stability due to gyroscopic effects. The following discussion of system stability, based upon (28) and (29), has not been published previously.

The first stability margin plot of logarithmic decrement versus dimensionless rotational speed is shown in Figure 4. For this plot, the damping ratio $\zeta_{r, 0}$ was set to 0.7 . It is apparent that the effective damping is a strong function of the gyroscopics and the rotational speed. For a thin disk $(P=2)$

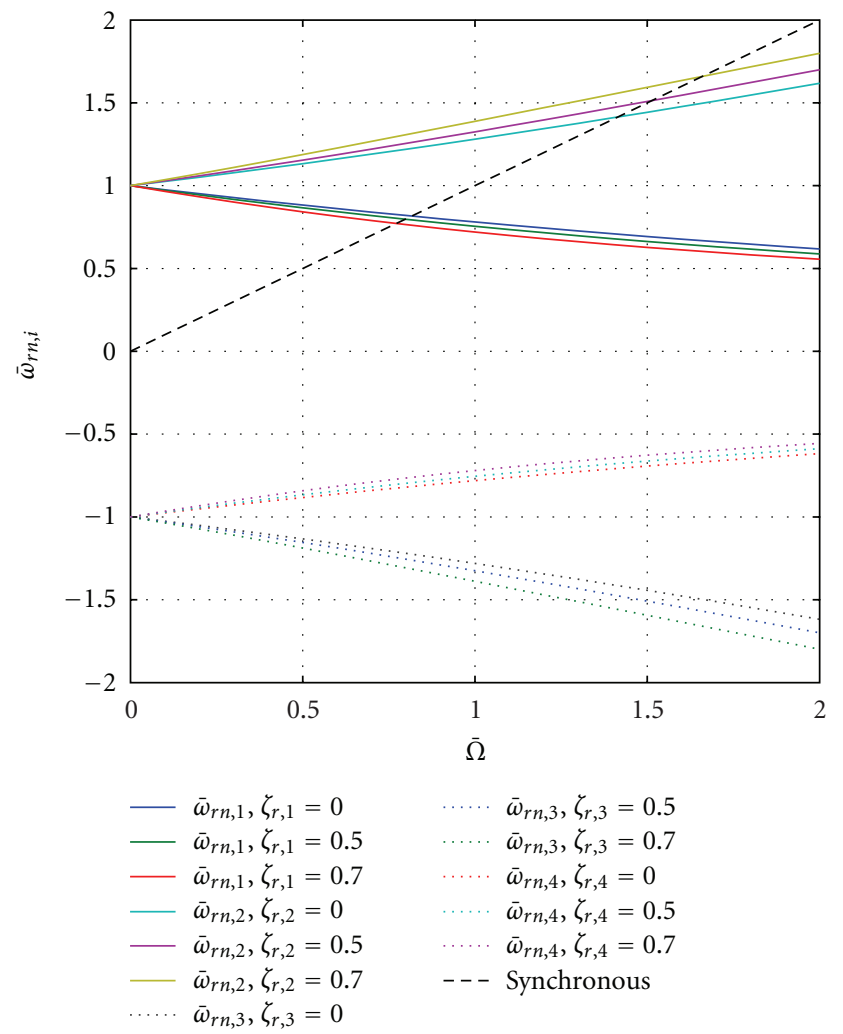

FIgURE 3: Undamped natural frequencies, various damping ratios, $P=0.5$.

rotating at twice the tilt natural frequency, the logarithmic decrement is 1.99 , which compares to a logarithmic decrement of 6.16 for zero rotational speed, or a reduction by a factor of 3 . As $P$ approaches zero, the effect on logarithmic decrement is less pronounced. For a typical value of $P=0.5$ for rigid rotor applications, and rotation at twice the tilt natural frequency, the logarithmic decrement is 4.64 . This is a 25 percent reduction compared to the zero-speed logarithmic decrement.

The second stability margin plot, Figure 5, shows similar results for a damping ratio of $\zeta_{r, 0}=0.5$. Again, considering the case of a thin disk where $P=2$, the logarithmic decrement is 1.41 when the disk is rotating at twice the tilt natural frequency, compared to a logarithmic decrement of 3.62 at zero rotational speed. The relative drop in logarithmic decrement is less than the $\zeta_{r, 0}=0.7$ case, since it only was reduced by a factor of 2.57 . With reduced $P$, the effect on logarithmic decrement is again reduced. For $P=0.5$, the logarithmic decrement for rotation at twice the tilt mode natural frequency is 3.05, which is a reduction of 16 percent compared to the zero-speed logarithmic decrement.

The third stability margin plot, Figure 6, shows the results for a damping ratio of $\zeta_{r, 0}=0.1$. A similar trend is observed when compared to the two previous damping ratios considered. For the thin disk rotating at twice the tilt natural frequency, the logarithmic decrement is 0.28 , which compares to the zero-speed logarithmic decrement of 0.63 . 


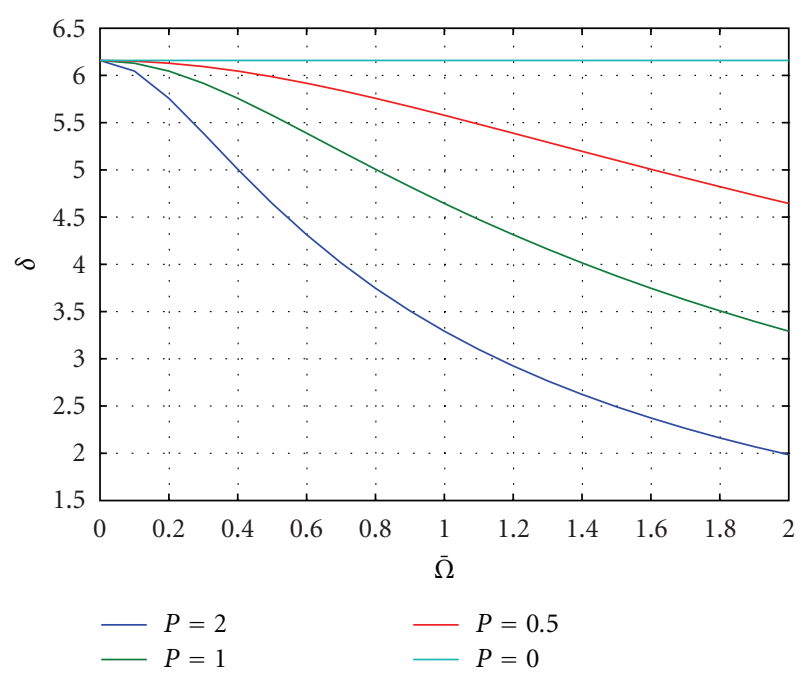

Figure 4: Log decrement as a function of dimensionless running speed, $\zeta_{r, 0}=0.7$.

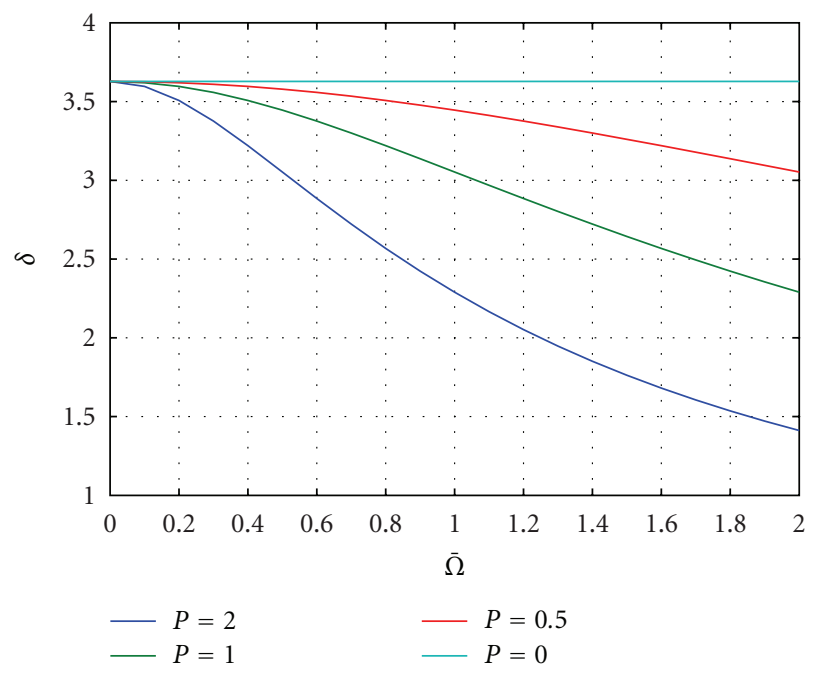

Figure 5: Log decrement as a function of dimensionless running speed, $\zeta_{r, 0}=0.5$.

The stability margin is reduced by a factor of 2.25 in this case. As with the previous cases, the effect is less pronounced as the inertia ratio $P$ approaches zero. For $P=0.5$, the logarithmic decrement for rotation at twice the tilt critical speed is 0.56 , which is a 12 percent reduction compared to the zero-speed logarithmic decrement.

The fourth stability margin plot, Figure 7 , shows the results for a damping ratio of $\zeta_{r, 0}=0.05$. The logarithmic decrement at zero rotation is 0.31 for the thin disc. When the thin disc is rotating at twice the tilt mode critical speed, the logarithmic decrement is reduced by a factor of 2.21 to 0.14 . The effect is most pronounced when the rotor approaches a thin disc and becomes less prevalent as the rotor approaches a long cylinder, implying that $P$ is approaching zero. For $P=0.5$, the logarithmic decrement for rotation at twice

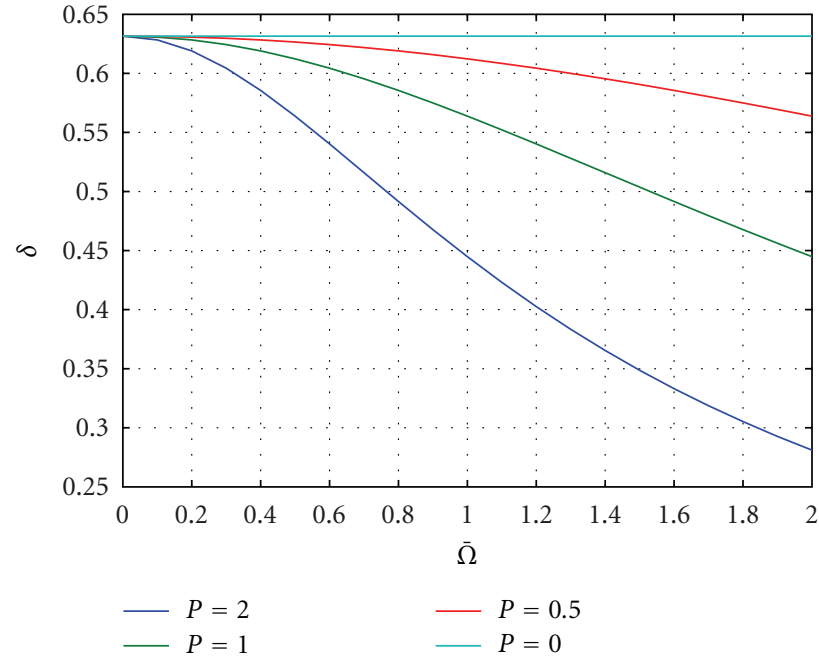

Figure 6: Log Decrement as a Function of Dimensionless Running Speed, $\zeta_{r, 0}=0.1$.

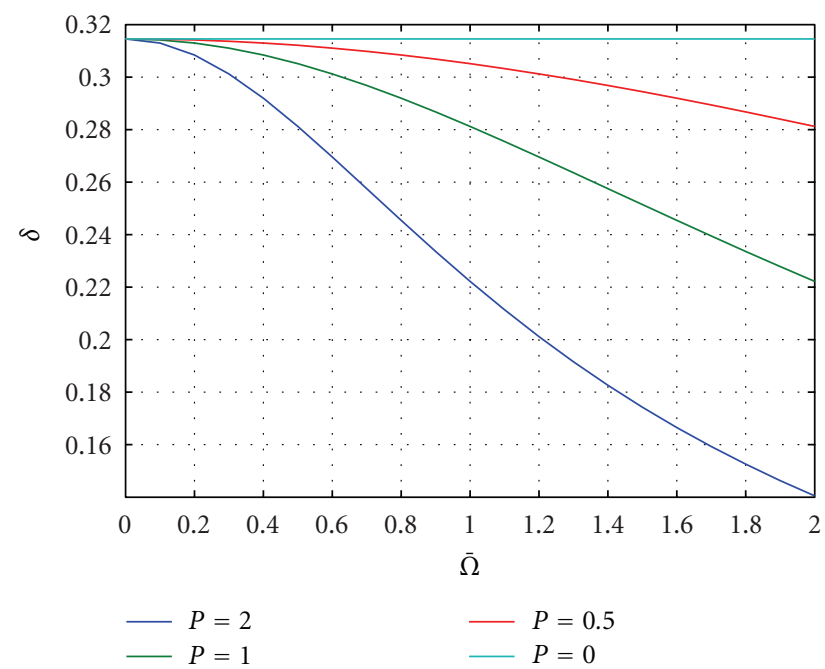

Figure 7: Log decrement as a function of dimensionless running speed, $\zeta_{r, 0}=0.05$.

the tilt mode natural frequency is 0.28 , or a reduction of 10 percent compared to the zero-speed logarithmic decrement.

\section{Conclusions}

In the present work, an analytical dimensionless damped eigenvalue solution was developed for a rigid rotor supported in AMBs using modal PD control. The tilt mode analytical solution is completely nondimensional and is expressed solely in terms of three dimensionless parameters: dimensionless rotational speed, zero-speed damping ratio, and the ratio of polar mass moment of inertia to transverse mass moment of inertia. The translating modes are not affected by the system gyroscopics and yield a typical underdamped eigenvalue solution. The tilt modes are affected by the gyroscopics and the 
expressions for the damped eigenvalues become relatively complex functions.

To understand the tilt mode functions in greater detail, Campbell diagrams are presented. The Campbell diagram of the undamped tilt mode eigenvalues shows the effect of gyroscopics on the undamped natural frequencies, and the effects of mode splitting. Based on the mode split, only some of the modes can ever be excited by synchronous excitations. The number of modes that can be excited by synchronous excitations is a function of the moment of inertia ratio $P$ and the unbalance distribution. However, all the forward and backward modes within the controller bandwidth can be excited by the AMBs [22], making it important to consider them in controller design. Another Campbell diagram, considering the undamped natural frequency for different damping ratios and $P=0.5$, shows that the undamped natural frequency is relatively insensitive to damping ratio, with a maximum change of about 5 percent from the undamped case to $\zeta_{r, 0}=0.7$.

The effect of the gyroscopics on the real part of the eigenvalue, which directly affects the rotor stability, was also explored. The modal PD controller does not explicitly have an $x-y$ coupling designed to control the gyroscopic forces. Plots of rotor stability as a function of nondimensional running speed show the effect of the gyroscopics on the stability margin. For nondimensional running speeds of 2 and $P=2$, the effect is a reduction in logarithmic decrement by a factor of 2.25-3 compared to the zero-speed logarithmic decrement, depending on damping ratio. This effect is a strong function of the moment of inertia ratio $P$ and disappears when $P=0$ or is near $P=0$ for long thin rotors. It is interesting to note that while the gyroscopic effect is not destabilizing [18], the gyroscopics can reduce the effective damping of the system. If additional destabilizing mechanisms are present in the system, such as radial seals, then there is the potential for an unstable rotor that would not be apparent from zero-speed damping ratios.

Many industrial AMB rotors have a $P$ ratio of 0.5 or less. The change in logarithmic decrement was about 25 percent for a damping ration of $\zeta_{r, 0}=0.7$ and became less pronounced for lower zero-speed damping ratios. This change in logarithmic decrement coupled with the relative insensitivity of the rotor natural frequencies to damping indicate that the gyroscopics can then be treated as an uncertainty when modern control theories such as $H_{\infty}$ or $\mu$-synthesis are applied to the control problem for rigid rotors supported by AMBs.

The development of a general solution to the rigid rotor supported in active magnetic bearings also has the benefit of familiarizing mechanical engineers with the basics of control algorithms for rotating systems and gives additional physical insight. The solution also allows for additional study of the effect of other destabilizing mechanisms that may be present in $\mathrm{AMB}$ rigid-rotor systems.

The effects studied here are generally quite different when the rotor is flexible. It is strongly recommended that model based controllers such as $H_{\infty}$ or $\mu$ synthesis be used for flexible rotors.

\section{Acknowledgment}

The funding and interest of the Rotating Machinery and Controls Laboratory at the University of Virginia is gratefully acknowledged.

\section{References}

[1] P. E. Allaire, E. H. Maslen, and R. R. Humphris, "Magnetic bearings," in CRC Handbook of Lubrication Technology, CRC Press, Boca Raton, Fla, USA, 1994.

[2] Z. Gosiewski and Falkowski, "Two-axial gyroscope with magnetically supported rotor," in Proceedings of the International Symposium on Magnetic Bearings, F. Matsumura, Ed., pp. 6570, Kanazawa, Japan, 1996.

[3] F. Matsumura, M. Fujita, and K. Okawa, "Modeling and control of magnetic bearing systems achieving a rotation around the axis of rotation," in Proceedings of the 2nd International Symposium on Magnetic Bearings, T. Higuchi, Ed., pp. 273-280, Tokyo, Japan, 1990.

[4] T. Mizuno and T. Higuchi, "Design of magnetic bearing controller based upon disturbance rejection," in Proceedings of the 2nd International Symposium on Magnetic Bearings, T. Higuchi, Ed., pp. 281-288, 1990.

[5] J. Kim and D. C. Han, "Robust centralized controller design for a rotor system supported by magnetic bearings," in Proceedings of the 3rd International Symposium on Magnetic Bearings, P. Allaire, Ed., pp. 527-536, 1992.

[6] J. Lottin, J. C. Ponsart, and P. Mouille, "Non-linear control of active magnetic bearings: digital implementation," in Proceedings of the 5th International Symposium on Magnetic Bearings, F. Matsumura, Ed., pp. 77-88, Kanazawa, Japan, 1996.

[7] J. Cao, L. Yu, and Y. Xie, "Study on a magnetic levitation flywheel energy storage device," in Proceedings of the 7th International Symposium on Magnetic Bearings, G. Schweitzer, Ed., pp. 69-74, Zurich, Switzerland, 2000.

[8] Y. S. Ho, P. Jiang, and L. Yu, "Dynamics of a rigid rotormagnetic bearing system equipped with a thrust magnetic bearing," in Proceedings of the 7th Symposium on Magnetic Bearings, G. Schweitzerpages, Ed., pp. 583-588, Zurich, Switzerland, 2000.

[9] L. Zhao, K. Zhang, R. Zhu, and H. Zhao, "Experimental research on a momentum wheel suspended by active magnetic bearings," in Proceedings of the 8th International Symposium on Magnetic Bearings, Y. Okada and K. Nonami, Eds., pp. 605609, Mito, Japan, 2002.

[10] A. Pilat, "Feedback linearization control of AMB system," in Proceedings of the 8th International Symposium on Magnetic Bearings, Y. Okada and K. Nonami, Eds., pp. 465-470, Mito, Japan, 2002.

[11] J. V. Löwis and J. Rudolph, "Adaptive inertial autocentering of a rigid rotor with unknown imbalance supported by active magnetic bearings," in Proceedings of the 7th Symposium on Magnetic Bearings, G. Schweitzer, Ed., pp. 583-588, Zurich, Switzerland, 2000.

[12] L. Li, T. Shinshi, X. Zhang, and A. Shimokohbe, "A simple method for rotation about the inertial axis of a rigid rotor," in Proceedings of the 8th International Symposium on Magnetic Bearings, Y. Okada and K. Nonami, Eds., pp. 405-410, Mito, Japan, 2002.

[13] H. Jeon, C. Lee, and S. Kim, "Dynamic analysis and control of rigid rotor supported by noncollocated active magnetic 
bearings," in Proceedings of the 8th International Conference on Rotordynamics, pp. 116-122, Seoul, Rebublic of Korea, 2010.

[14] Z. Gosiewski, "Analytical analysis of MIMO magnetic bearingrotor system," in Proceedings of the 8th International Conference on Rotordynamics, pp. 746-753, Seoul, Rebublic of Korea, 2010.

[15] R. Larsonneur, "Control of the rigid rotor in AMBs," in Magnetic Bearings - Theory, Design, and Application to Rotating Machinery, G. Schweitzer and E. Maslen, Eds., chapter 8, pp. 191-228, Springer, Berlin, Germany, 2009.

[16] P. E. Allaire, T. W. Dimond, and Z. Lin, "Rotordynamic analysis of machines on active magnetic bearings: comparison to fluid film bearing machines and vibration specifications," International Journal of Modelling, Identification and Control, vol. 7, no. 1, pp. 57-66, 2009.

[17] J. M. Vance, Rotordynamics of Turbomachinery, John Wiley \& Sons, New York, NY, USA, 1988.

[18] T. Yamamoto and Y. Ishida, Linear and Nonlinear Rotordynamics - A Modern Treatment with Applications, John Wiley \& Sons, New York, NY, USA, 2001.

[19] G. Genta, Dynamics of Rotating Systems, Springer, New York, NY, USA, 2005.

[20] A. Mostowski and M. Stark, "Properties of complex numbers," in Introduction to Higher Algebra, I. N. Sneddon, M. Stark, and S. Ulam, Eds., vol. 37 of International Series of Monographs in Pure and Applied Mathematics, chapter 4, pp. 80-93, Pergamom Press, Oxford, UK, 1964.

[21] S. Rabinowitz, "How to find the square root of a complex number," Mathematics and Informatics Quarterly, vol. 3, pp. 54-56, 1993.

[22] J. Jumonville, "A new method of accurately identifying the location and movement of forward and backward bending modes using magnetically suspended rotor systems," in Proceedings of the 37th Turbomachinery Symposium, pp. 67-71, Texas A\&M University Press, 2008. 

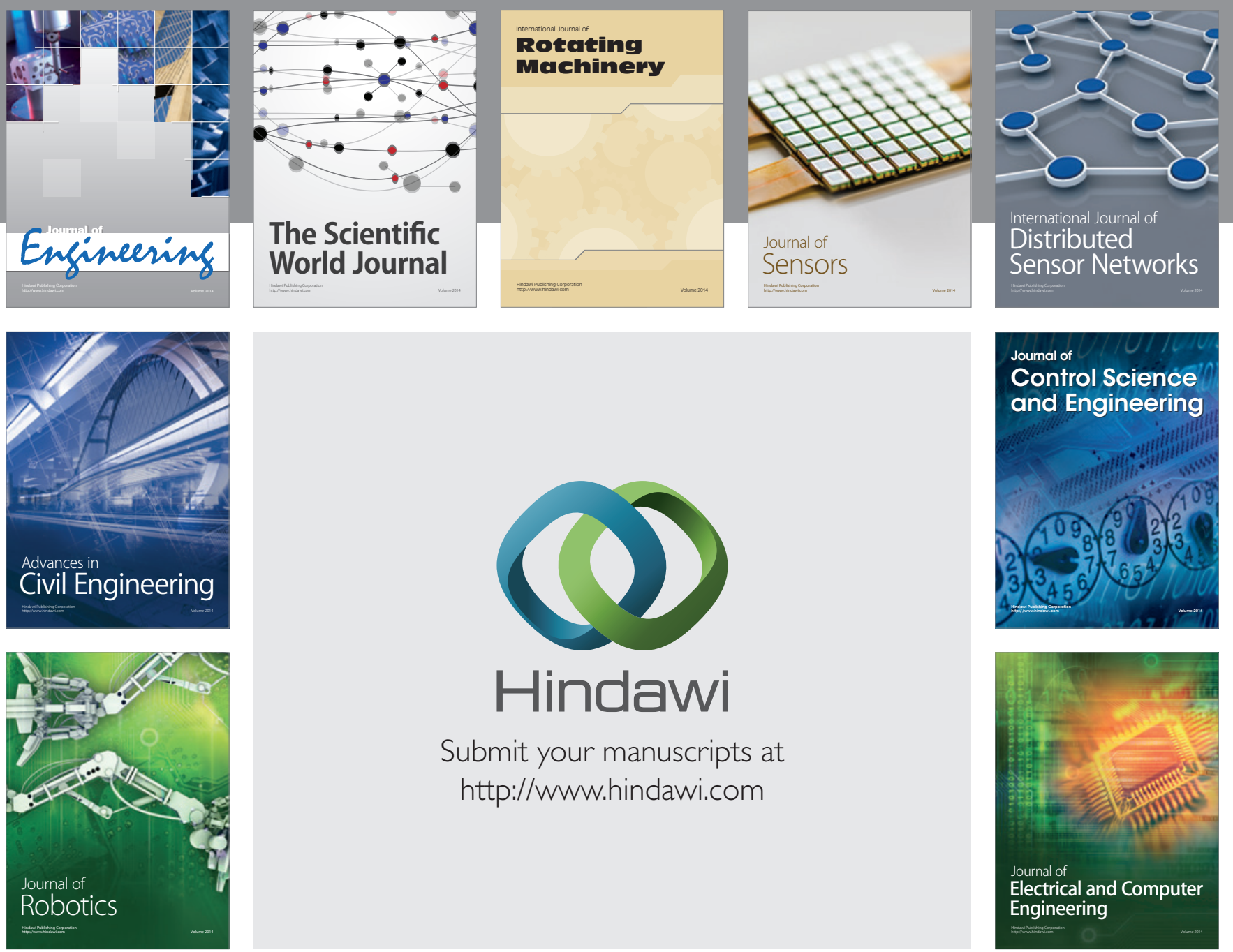

Submit your manuscripts at

http://www.hindawi.com
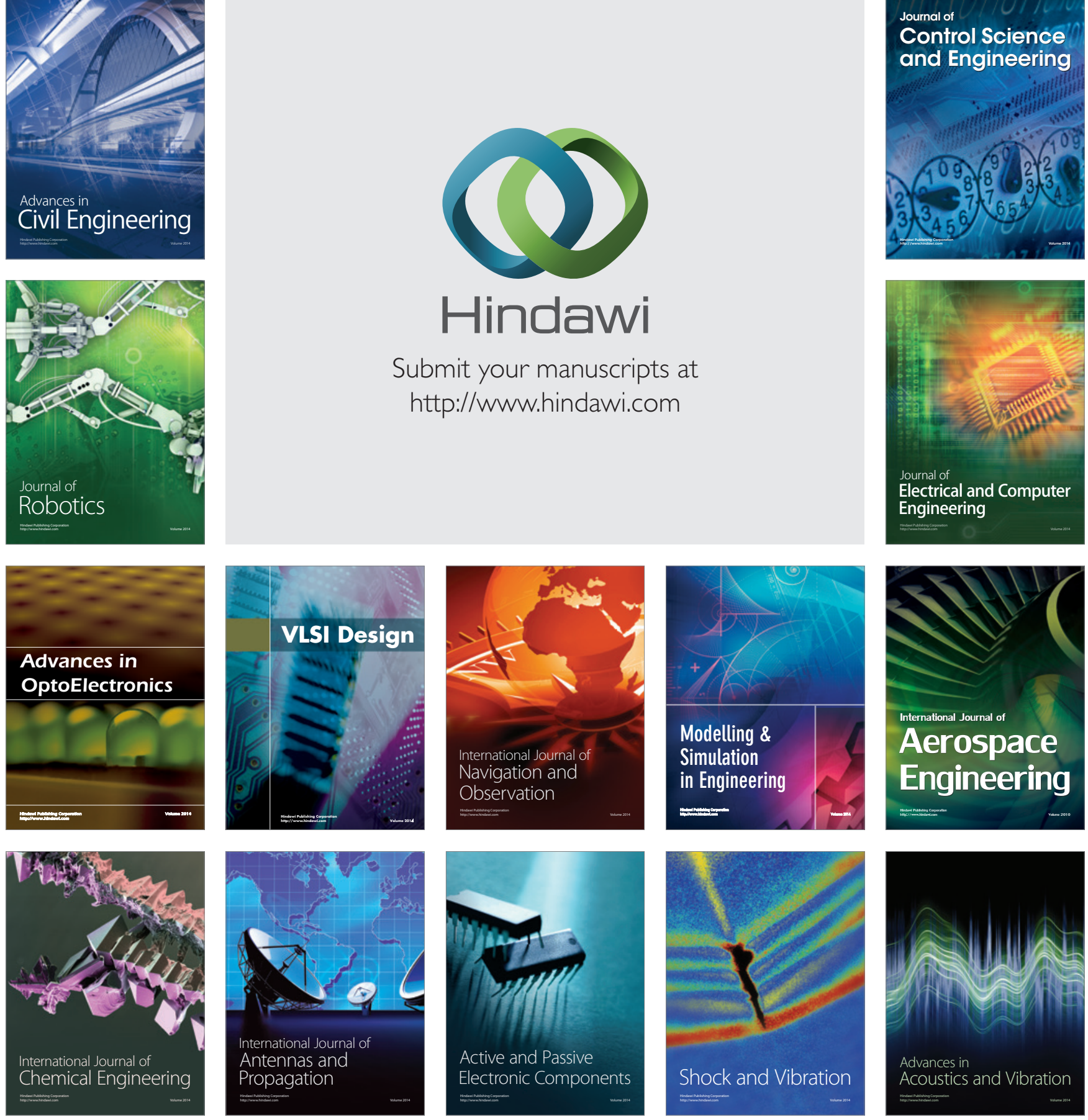